\title{
Evaluation of peptide fractionation and native digestion as two novel sample preparation workflows to improve HCP characterization by LC-MS/MS
}

Regina Kufer*1, Markus Haindl ${ }^{1}$, Harald Wegele ${ }^{1}$, Stefanie Wohlrab ${ }^{1}$

\section{Supporting Information}

\section{Table of contents}

- Figure 1-S: Number of peptides identified for 8 protein standard spiked into $\mathrm{mAb1}$ at $5 \mathrm{ppm}$ level (n=7)

- Figure 2-S: HCP identification of 8 protein standard at $5 \mathrm{ppm}$ level as replicate of Figure 1A measured on a Orbitrap Fusion $(n=3)$

- Figure 3-S: Individual plots of \% increase in number of peptides for every spiked HCP of 8 protein standard

- Figure 4-S: Total ion chromatograms comparing non fractionated to native digestion and $2 \mathrm{~h}$ and overnight digestion time

- Figure 5-S: method control sample analyzed over time

- Figure 6-S: Absolute numbers of identified HCPs in protein A elution pools of 5 different mAbs

- $\quad$ Figure 7-S: Venn diagrams of fractionated pellet digestion for all $5 \mathrm{mAbs}$

- Figure 8-S: Correlation of amount of starting material and pellet size 


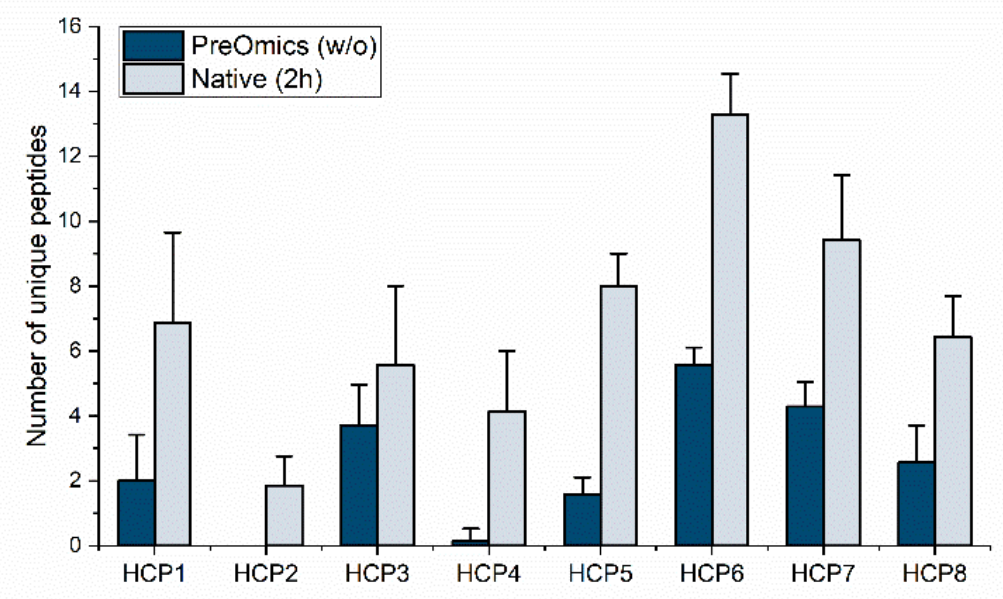

Figure 1-S. Replicates referring to Figure 1A. Comparing number of unique peptides identified in PreOmics non-fractionated and native digested 5 ppm 8 protein standard samples. Error bars show standard deviation of 7 replicates.

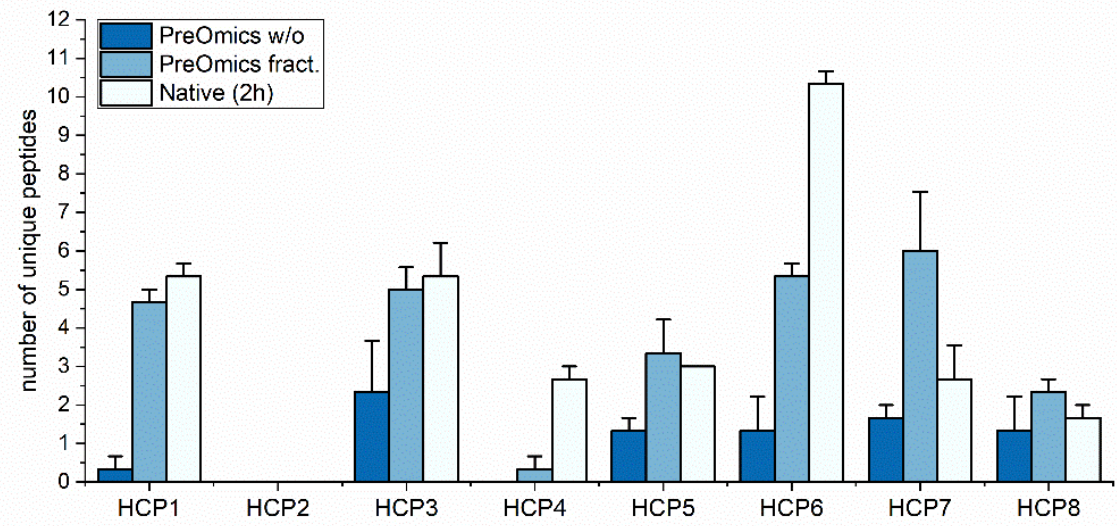

Figure 2-S. HCP identification of 8 protein standard at $5 \mathrm{ppm}$ level as replicate of Figure 1A measured on a Orbitrap Fusion ETD in three process replicates. Error bars show standard error. 
$\square$ PreOmics fract. $\square$ Native (2h) $\square$ Native (oN)
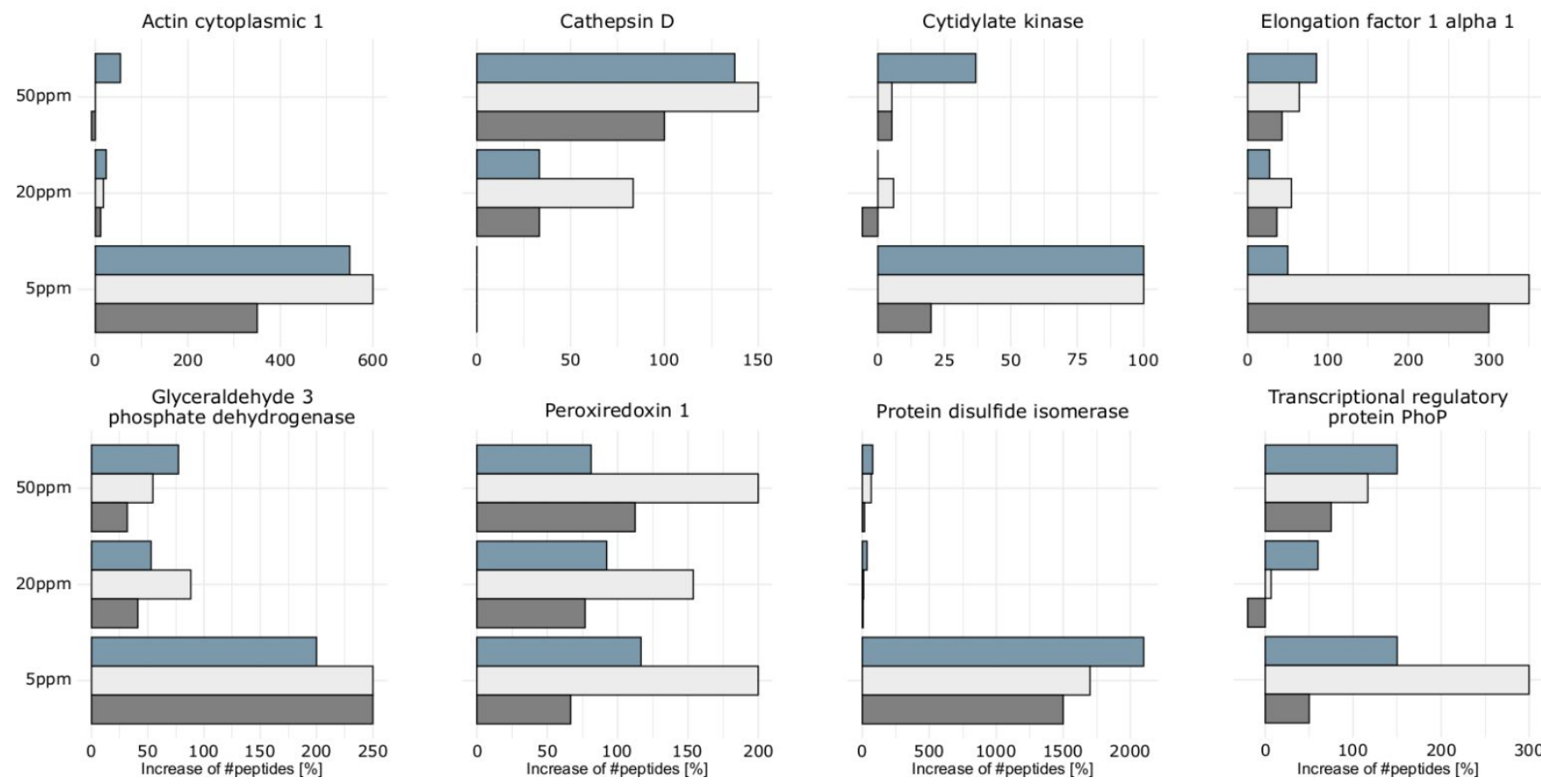

Protein disulfide isomerase

Transcriptional regulatory
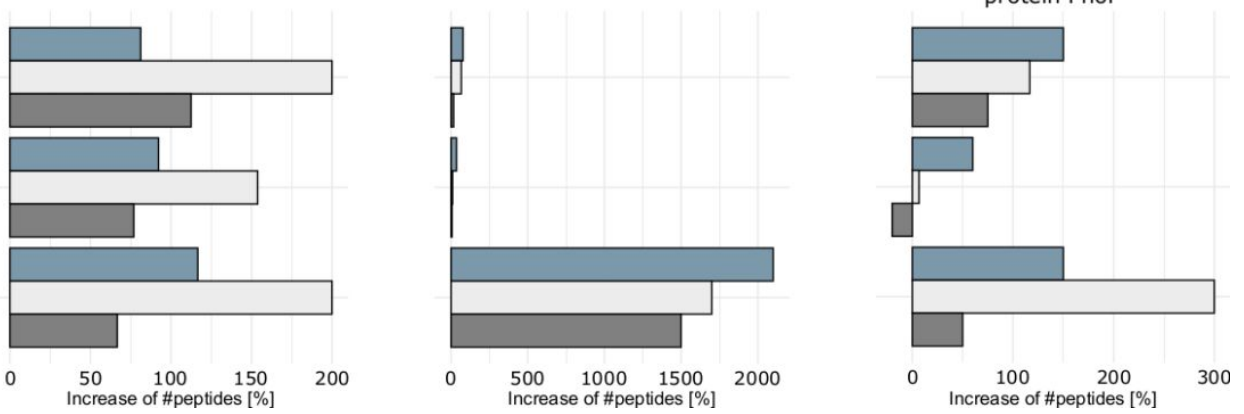

Figure 3-S. Detailed information on Figure 1B. Increase [\%] of detected unique peptides relative to non-fractionated digestion at 5, 20 and $50 \mathrm{ppm}$ level separately plotted for every HCP spiked. The increase was calculated by the formula (\# peptides (novel sample prep) / \# peptides $($ PreOmics w/o) - 1) * 100. Thus, positive values reflect an actual increase in number of peptides identified for a certain protein.
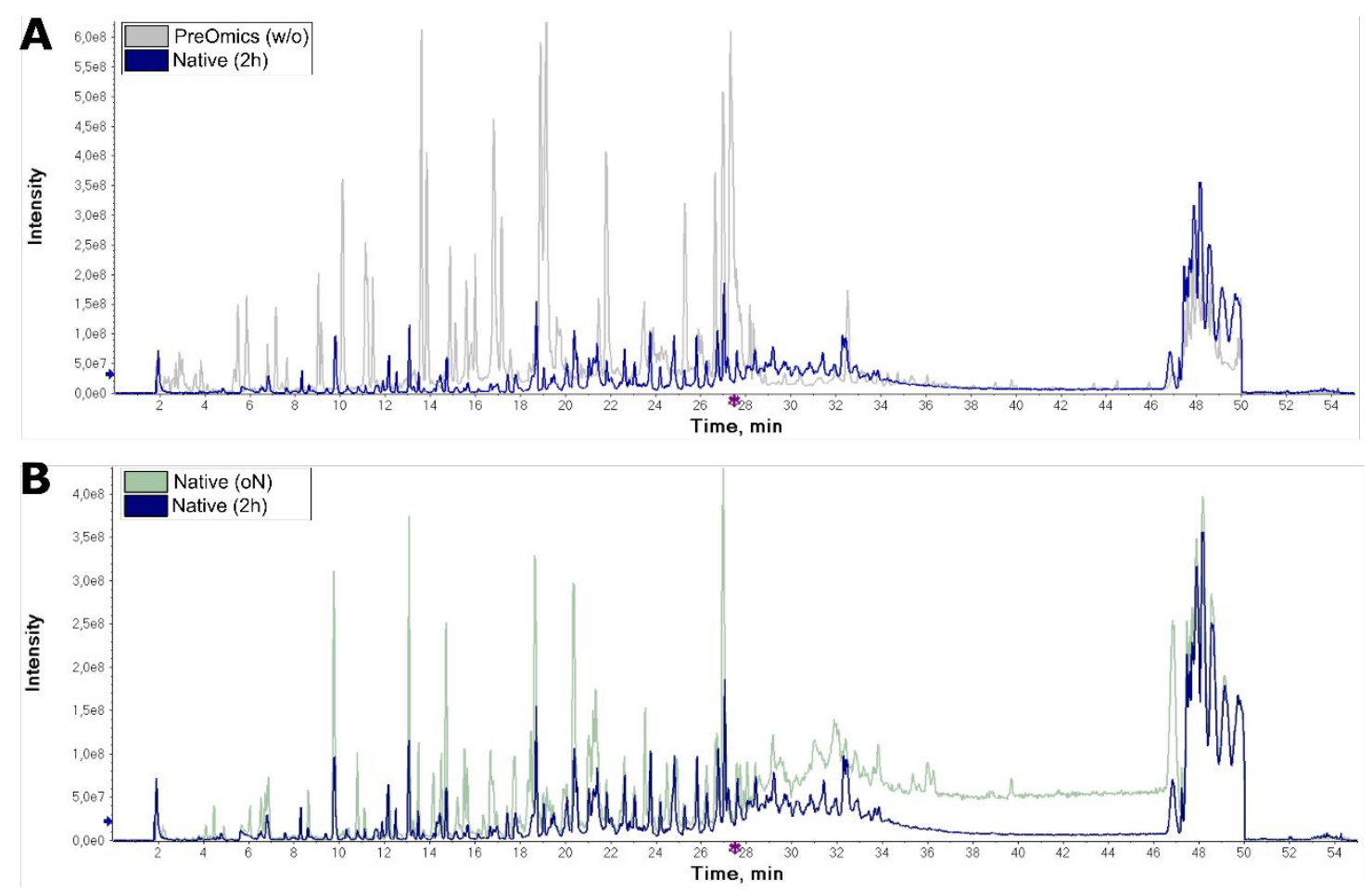

Figure 4-S. A) Total ion chromatogram (TIC) overlay of the 8 protein standard spiked into mAb1 at 5 ppm level prepared with PreOmics non-fractionated and native digestion (2h) showing less high intense product peaks. B) TIC overlay of the 8 protein standard spiked into $\mathrm{mAb} 1$ at $5 \mathrm{ppm}$ level comparing overnight and $2 \mathrm{~h}$ native digestion. 


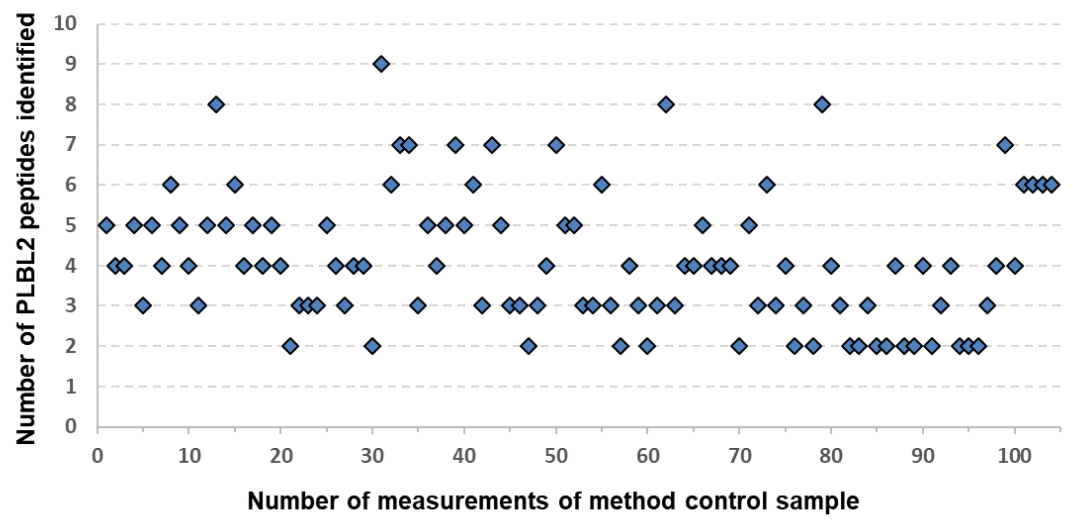

Figure 5-S. A) 50 ppm PLBL2 was spiked into antibody product and analyzed as method control sample in 104 experiments over 29 months. PLBL2 was reliably identified with our acceptance criteria of at least 2 unique peptides @ 95\% confidence. In the whole test session the number of unique PLBL2 peptides identified ranged between 2 and 9.

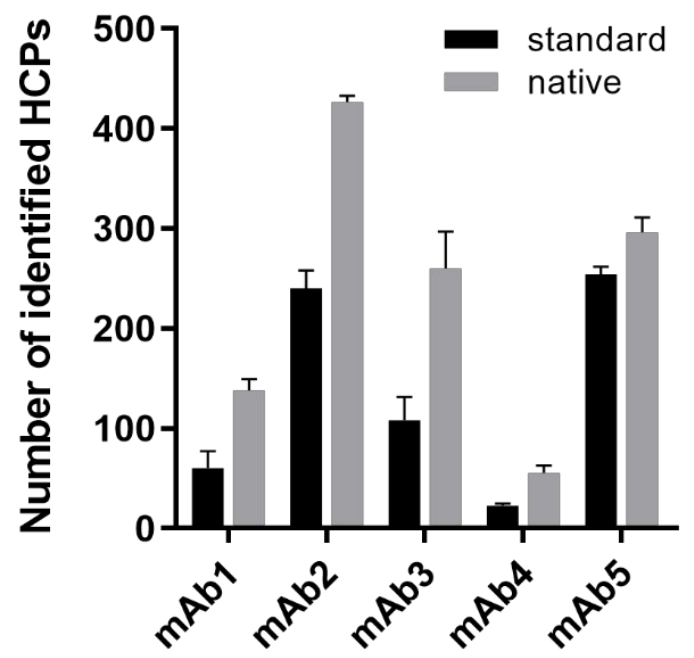

Figure 6-S. Absolute numbers of identified HCPs in protein A elution pools of 5 different mAbs comparing standard and native digestion. Error bar shows the standard deviation of the number of positively identified proteins measured in 3 replicates.

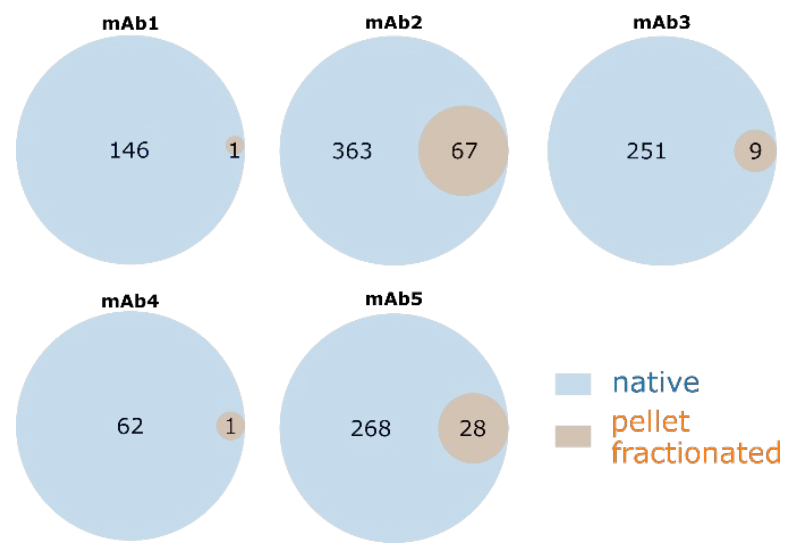

Figure 7-S. Detailed information to Figure 3. Venn diagrams of fractionated pellet digestion for all $5 \mathrm{mAbs}$. 


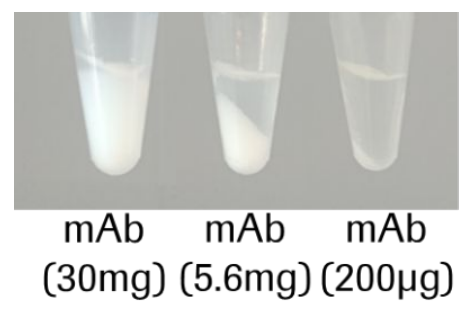

Figure 8-S. We observed an increase in pellet size when a higher amount of starting material was subjected to native digestion. In our study we used $200 \mu \mathrm{g}$ protein sample. 